\title{
Rate an Leukämien und kolorektalen Karzinomen etwas erhöht
}

Fragestellung: Erhöht die Therapie mit Mitoxantron bei Patienten mit Multipler Sklerose (MS) das Risiko für Krebserkrankungen?

Hintergrund: Mitoxantron ist vor gut 15 Jahren für die Behandlung der sekundär chronisch-progredienten MS oder auch der hochaktiven schubförmigen MS zugelassen worden. Seitdem, insbesondere vor der Zulassung der modernen monoklonalen Antikörper, sind Tausende von Patienten weltweit mit Mitoxantron behandelt worden. Immer wieder gab es Hinweise darauf, dass Mitoxantron bei der langfristigen Gabe nicht nur kardiologische Probleme verursachen kann, sondern auch langfristig die Rate an Malignomen erhöht. In dieser retrospektiven Studie ist der Frage nachgegangen worden, wie viele mit Mitoxantron behandelte MS-Patienten Krebserkrankungen entwickelt haben. Diese Daten sind dann mit den Prävalenzen einzelner Krebserkrankungen in der allgemeinen Bevölkerung statistisch abgeglichen worden.

Patienten und Methoden: Diese retrospektive Studie schloss alle MS-Patienten ein, die zwischen den Jahren 1994 und 2007 in der Universitätsklinik Würzburg mit Mitoxantron behandelt wurden. Die Patienten wurden bis zum Jahr 2010 im Hinblick auf die Entwicklung von Malignomen sowie - soweit verstorben - hinsichtlich der Todes-

Burrmann M, Seuffert L, Mäder $\mathrm{U}$ et al. Malignancies after mitoxantrone for multiple sclerosis. Neurology 2016; 86: 1 -5 ursache weiter verfolgt. Die Malignomrate wurde dann mit dem deutschen nationalen Krebsregister verglichen und im Hinblick auf die wichtigsten Begleitfaktoren wie Geschlecht, Alter, Dauer der Erkrankung und Jahr des Auftretens abgeglichen.

Ergebnisse: Insgesamt wurden 776 Patienten ausfindig gemacht, die auch weiter verfolgt werden konnten. Die mittlere Nachverfolgungszeit betrug 8,7 Jahre. Dies entsprach 6.222 Patientenjahren. Die mittlere verwendete Mitoxantron-Dosis betrug 79 $\mathrm{mg} / \mathrm{m}^{2}$ Körperoberfläche.

Insgesamt wurden 37 Patienten (5,5\%) mit einem Malignom diagnostiziert, das nach der ersten Mitoxantron-Infusion aufgetreten war. An Krebsentitäten zeigten sich Mammakarzinome, kolorektale Karzinome, akute myeloische Leukämien sowie andere Erkrankungen. Die standardisierte Inzidenzrate der kolorektalen Karzinome betrug 2,98, die der akuten myeloischen Leukämie 10,44. Die beiden Werte waren im Vergleich zu den Inzidenzraten im deutschen Krebsregister leicht erhöht. Für alle anderen Malignitäten inklusive Mammakarzinomen zeigten sich keine erhöhten Werte.

Die multivariante Cox-Regressionsanalyse ergab als spezifische Risikofaktoren nur ein höheres Alter bei Behandlungsbeginn, wobei weder die kumulative Dosis von unter- oder über $75 \mathrm{mg} / \mathrm{m}^{2}$ eine Zusatzbehandlung mit anderen immunsuppressiven Substanzen oder das Geschlecht als Risikofaktoren identifiziert wurden.

Schlussfolgerungen: Unter der langjährigen Gabe von Mitoxantron erhöhen sich leicht die Raten an kolorektalen Karzinomen sowie akuter myeloischer Leukämien. Patienten nach einer Mitoxantron-Behandlung sollten daher aufmerksam überwacht werden.

\section{- Kommentar von Volker Limmroth, Köln \\ Die Überwachung der Patienten ist wichtig}

Die Ergebnisse dieser retrospektiven Studie sind nicht überraschend. Die gute Nachricht in dieser Studie ist dennoch, dass die Malignomraten, wenn überhaupt nur geringgradig erhöht sind und das scheinbar auch nur für zwei Entitäten. Die Daten zeigen aber auch, dass man Patienten nach Mitoxantron-Therapie regelmäßig überwachen sollte, zumindest im Hinblick auf das Blutbild. Ob sich hieraus eine höhere Frequenz von Koloskopien ergeben sollte, muss noch offen bleiben. In jedem Fall sollte man diese Patientengruppe zu Vorsorgeuntersuchungen ermutigen.

Im Hinblick auf die Tatsache, dass nur Patienten bis 2007 eingeschlossen worden sind, die dann bis zum Jahr 2010 nachverfolgt wurden, hätte man sich die Veröffentlichung dieser Daten natürlich deutlich früher gewünscht. Denn im Hinblick auf die neuen Therapiemöglichkeiten wird die Zahl von neuen
Mitoxantron-Patienten sicher immer geringer werden, doch die Überwachung der bisher behandelten Patienten ist offensichtlich wichtig.

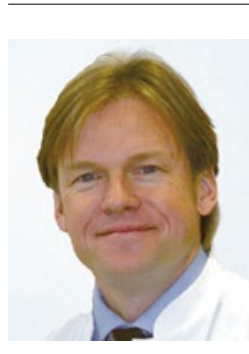

Prof. Dr. med. Volker Limmroth, Köln-Merheim

Chefarzt der Klinik für Neurologie und Palliativmedizin Köln-Merheim E-Mail: LimmrothV@kliniken-koeln.de 